\title{
Fatty Acid Synthesis Pathway Genetic Variants and Clinical Outcome of Non-Small Cell Lung Cancer Patients after Surgery
}

\author{
Xin Jin ${ }^{1,2 \&}$, Ke-Jin Zhang ${ }^{3,4 \&}$, Xu Guo $^{2}$, Ronald Myers ${ }^{3}$, Zhong Ye ${ }^{3}$, Zhi-Pei Zhang ${ }^{5}$, \\ Xiao-Fei $\mathrm{Li}^{5}$, Hu-Shan Yang ${ }^{3}$, Jin-Liang Xing ${ }^{2 *}$
}

\begin{abstract}
Over-expression of de novo lipogenesis (DNL) genes is associated with the prognosis of various types of cancers. However, the effects of single nucleotide polymorphisms (SNPs) in these genes on recurrence and survival of non-small cell lung cancer (NSCLC) patients after surgery are still unknown. In this study, a total of 500 NSCLC patients who underwent surgery treatment were included. Eight SNPs in 3 genes (ACACA, FASN and ACLY) of the DNL pathway were examined using the Sequenom iPLEX genotyping system. Multivariate Cox proportional hazards regression and Kaplan-Meier curves were used to analyze the association of SNPs with patient survival and tumour recurrence. We found that two SNPs in the FASN gene were significantly associated with the recurrence of NSCLC. SNP rs 4246444 had a significant association with lung cancer recurrence under additive model (hazard ratio $[\mathrm{HR}], 0.82 ; 95 \%$ confidence interval $[95 \% \mathrm{CI}], 0.67-1.00 ; p=0.05$ ). Under the dominant model, rs4485435 exhibited a significant association with recurrence (HR, 0.75; 95\% CI, 0.56-1.01; $p=0.05)$. Additionally, SNP rs9912300 in ACLY gene was significantly associated with overall survival in lung cancer patients $(\mathrm{HR}, 1.41 ; 95 \% \mathrm{CI}, 1.02-1.94, p=0.04)$ under the dominant model. Further cumulative effect analysis showed moderate dose-dependent effects of unfavorable SNPs on both survival and recurrence. Our data suggest that the SNPs in DNL genes may serve as independent prognostic markers for NSCLC patients after surgery.
\end{abstract}

Keywords: De novo lipogenesis -FASN -non-small cell lung cancer - prognosis - single nucleotide polymorphisms

Asian Pac J Cancer Prev, 15 (17), 7097-7103

\section{Introduction}

Lung cancer is the leading cause of cancer-related mortality worldwide, and non-small cell lung cancer (NSCLC) accounts for $80 \%$ of all types of lung cancer (Jemal et al., 2011). Despite advances in NSCLC treatment, the five-year overall survival (OS) rate of NSCLC remains less than 15\% (Goldstraw et al., 2011). Radical resection is generally considered as the most effective treatment for early stage NSCLC. However, a large proportion of patients die of local recurrence or distant metastasis within several years after surgery. Therefore, there is a critical need for prognostic biomarkers that will improve the clinical management of patients with NSCLC.

Altered metabolism is one of the important features of cancer (Cantor and Sabatini, 2012). Initially, Otto Warburg observed the enhanced anaerobic glycolysis in cancer (Warburg, 1956). Most of the glycolytic endproduct, pyruvate, is converted to lactate, whereas some is converted to acetyl-CoA that is in turn used for de novo fatty-acid synthesis, termed de novo lipogenesis (DNL) (Swinnen et al., 2006). Highly proliferating cancer cells need to synthesize fatty acids de novo to continually provide lipids for energy production, cell membrane regeneration and lipid modification of protein. In addition, fatty acids and their derivatives are also important signaling molecules that may affect many fundamental cell processes, including cellular survival, proliferation, migration, invasion, angiogenesis and therapy resistance. DNL involves three rate-limiting enzymes, i.e. ATP citrate lyase (ACLY), acetyl-CoA carboxylase $\alpha$ (ACACA) and fatty-acid synthase (FASN). ACLY converts citrate into cytosolic acetyl-CoA, which is transformed into malonylCoA by the catalysis of ACACA. The malonyl-CoA product is further converted by FASN to long-chain fatty acids. In cancer cells, DNL is commonly elevated and the supply of cellular fatty acid is highly dependent on the de novo synthesis. Therefore, deregulated de novo fatty-acid synthesis directly leads to cellular fatty-acid accumulation and affects fundamental cellular processes, including 
signal transduction and gene expression.

Numerous studies have shown the over-expression of DNL enzymes in various human epithelial cancers, including prostate, ovary, colon, lung, endometrial and stomach cancers (Kuhajda, 2000). Moreover, several reports have shown that FASN and related DNL enzymes play important roles in tumor cell survival at multiple levels (Chajes et al., 2006; Migita et al., 2009; Li et al., 2012a; Lin et al., 2013). In addition, over-expression of FASN or ACLY indicates poor prognosis of patients with malignancies, including breast cancer, nasopharyngeal carcinoma, colorectal, ovarian and prostate cancer, as well as NSCLC (Alo et al., 1996; Shurbaji et al., 1996; Ogino et al., 2008; Cerne et al., 2010; Rahman et al., 2012; Wang et al., 2012; Kao et al., 2013) .

Single nucleotide polymorphism (SNP), a common typical genomic variation, can be used as a stable biomarker of genetic background to predict the risk, therapeutic response and prognosis of cancer (Laing et al., 2011; Liu et al, 2012). Several studies have also discussed the relationship between polymorphisms of DNL genes and development and prognosis of cancers (Nguyen et al., 2010; Eggert et al., 2012). However, no studies have focused on the association between polymorphisms of ACLY, ACC and FASN and NSCLC prognosis until now. Therefore, we assessed the effects of eight SNPs in these three genes on the post-operation recurrence and survival in a cohort of 500 Chinese NSCLC patients.

\section{Materials and Methods}

\section{Ethics statement}

The study was approved by the Ethical Committee of the Fourth Military Medical University and all study procedures were carried out in accordance with the ethical standards of the Helsinki Declaration. Written consent was obtained from each patient.

\section{Study population}

Between June 2009 and January 2012, NSCLC patients were enrolled at the Department of Thoracic Surgery Tangdu Hospital, the Fourth Military Medical University in Xi'an, China. Patients with newly diagnosed and histologically confirmed NSCLC were eligible for the enrollment. Patients with a history of other cancers were excluded. By January 2012, a total of 500 eligible NSCLC patients were recruited, with complete and validated demographic, clinical, and follow-up data. All patients received surgery within 2 months after diagnosis. The primary tumor was completely resected for all patients, and was confirmed by pathological review of the tumors after resection. No patient received neoadjuvant chemotherapy or radiotherapy.

\section{Epidemiologic and clinical data collection}

Demographic data were collected through in-person interviews at the time of initial visit or follow-up in the clinics, medical chart review, or consultation with the treating physicians by trained clinical research specialists. For data acquired from multiple sources, the research staff compared and validated the consistency of these data. The patients and/or family members were further contacted for verification if there were discrepancies. Individuals who smoked more than 100 cigarettes in their lifetime were defined as ever-smokers, otherwise as never-smokers. Tumor stage was assessed using the 7th American Joint Committee on Cancer (AJCC) TNM staging system. The follow-up information on recurrence and survival was updated at a 6-month interval through onsite interviews, direct calling, or medical chart review. The latest followups in this study were carried out in June 2012. Cancer recurrence was the primary endpoint measured in this study, which was defined as the occurrence of a tumor with the same histological type in the original organ (locoregional recurrence) or in a different organ (distant metastasis) after the resection of the primary tumor by surgery. The secondary endpoint was OS which was defined as time in months from the date of first diagnosis to death resulting from any cause.

\section{SNP genotyping}

Genomic DNA was obtained for each participant as described previously (Xing et al., 2011). Three DNL pathway genes (ACACA, FASN and ACLY) were included as candidate genes in this study to estimate the effects of their genetic polymorphisms on OS and recurrence in lung cancer patients. Referring to the databases of dbSNP (http://www.ncbi.nlm.nih.gov/project/SNP/) and the International HapMap Project (http://www.hapmap. ncbi.nlm.nih.gov/), a Web-Based Tagger tool (http:// broad.harvard.edu/mpg/tagger/) was applied to select the candidate SNPs as described previously (Zhou et al., 2012). Briefly, the strategy and criteria for the selection of tagger SNPs were as follows: a) located in target genes or their $1000 \mathrm{bp}$ flanking regions; b) potentially functional SNPs had priority; c) had minor allele frequency (MAF) $>5 \%$ in Chinese population (CHB). If there were multiple potentially functional SNPs within the same block (defined by the linkage coefficient $r 2>0.80$ ), only 1 tag SNP was included. Functional SNPs included missense SNPs in exons, SNPs in microRNA binding sites of 3' untranslated region, SNPs in the transcription factor binding site of the 5' flanking region, and SNPs in splice sites.

Based on the above criteria, eight SNPs were selected in this study, including two in transcription factor binding site sequences (rs11871275 and rs9912300), two missense SNPs (rs7211875 and rs2304497), two synonymous SNPs (rs1140616 and rs4485435), one

Table 1. Candidate SNPs in de Novo Lipogenesis Genes in this Study

\begin{tabular}{llll}
\hline Gene & SNPs & Allele & Functional analysis \\
\hline ACACA & rs1714987 & G/C & nsSNP/Splicing (ESE or ESS) \\
& rs11871275 & A/T & TFBS \\
& rs7211875 & T/C & Missense SNP Pro > Ser \\
ACLY & rs2304497 & T/G & Missense SNP Glu > Asp \\
& rs9912300 & T/G & TFBS \\
FASN & rs4246444 & T/G & NA \\
& rs1140616 & C/T & SynSNP \\
& rs4485435 & G/C & SynSNP \\
\hline
\end{tabular}

*Abbreviations: ACACA, Acetyl-CoA carboxylase-alpha; ACLY, ATP citrate lyase; FASN, Fatty acid synthetase; SNP, single nucleotide polymorphisms; nsSNP, nonsynonymous SNP; TFBS, transcription factor binding site; SynSNP, synonymous SNP; NA, not available 
splice site SNP (rs1714987), and one whose potential function is still unknown (rs4246444) (Table 1). Genotyping was performed with iPLEX (Sequenom, San Diego, CA) matrix-assisted laser desorption/ionization time-of-flight mass spectrometry system. Laboratory personnel conducting genotyping were blinded to patient information. Strict quality control measures were implemented during genotyping with more than $99 \%$ concordance in samples that were randomly selected to be genotyped in duplicate.

\section{Statistical analysis}

SAS software package (version 9.2; SAS Institute, Cary, NC, USA) was used for the aforementioned analyses. Three genetic models (additive, dominant and recessive) were applied to assess the association of single SNP with clinical outcome of lung cancer patients. The best-fitting model was defined as that with the smallest $P$ value and a lower trend $\mathrm{P}$ value. The $\mathrm{Q}$ value that represents a measure of significance in terms of the false discovery rate was used to adjust the significance level for individual SNPs in multiple tests correction. We calculated $\mathrm{Q}$ value by the Q-value package (version 1.0) implemented in the $\mathrm{R}$ software (Storey et al., 2004). Hazard ratios (HR) were estimated by multivariate Cox proportional hazards model, adjusting for age, gender, smoking status, TNM stage, differentiation, and adjuvant therapy. The cumulative effect of unfavorable genotypes identified in the single SNP analysis on recurrence and OS, was estimated by Cox model (Flynn, 2012). Kaplan-Meier curve and log-rank test were used to assess the differences in survival and time to recurrence between patients with different genotypes
(Dinse and Lagakos, 1982). All P-values in this study were two-sided, and $p<0.05$ was considered to be significant.

\section{Results}

Clinical characteristics and their impact on prognosis of NSCLC patients

A total of 500 NSCLC patients were included in this study, with a median age of 60 years (range, 27-86 years), and $110(22.0 \%)$ patients were female. More than 30.0\% of the patients $(n=154)$ had never smoked. The majority $(63.2 \%)$ of patients had moderately differentiated tumors, and $59.8 \%$ of the patients had tumor size larger than 5.0 $\mathrm{cm}$. Sixty-seven percent of the patients had received platinum-based adjuvant chemoradiotherapy after surgery. During the median follow-up of 15.6 months (ranging from 2 to 36.6 months), 165 (33.0\%) patients died of lung cancer, and more than $45.6 \%(n=226)$ patients developed recurrence. Cox regression analyses showed no significant effects of age, gender, smoking status and tumor size on OS and recurrence of NSCLC patients. TNM stage had significant associations with OS and recurrence in a doseresponse manner ( $p<0.01$ for both). Comparing to those without adjuvant therapy, patients who received adjuvant therapy had a significantly reduced death risk $(\mathrm{HR}=0.64$, 95\%CI 0.44-0.92, $p=0.02$ ) (Table 2).

\section{Association of single SNP with clinical outcomes of} NSCLC

We assessed the effects of eight SNPs in three DNL pathway genes (ACACA, FASN and ACLY) on the OS and recurrence in NSCLC patients using multivariate

Table 2. Distribution of Patients' Characteristics and Prognosis Analysis

\begin{tabular}{|c|c|c|c|c|c|c|c|}
\hline \multirow[t]{2}{*}{ Variables } & \multirow[t]{2}{*}{ Total } & \multicolumn{3}{|c|}{ Overall survival } & \multicolumn{3}{|c|}{ Recurrence-free survival } \\
\hline & & Death & HR (95\%CI) & $\mathrm{P}$ & Recurrence & HR (95\%CI) & $\mathrm{P}$ \\
\hline \multicolumn{8}{|l|}{ Age, year } \\
\hline$\leq 60$ & 242 & 81 & Ref. & & 119 & Ref. & \\
\hline$>60$ & 258 & 84 & $1.09(0.80-1.49)$ & 0.58 & 107 & $0.93(0.71-1.21)$ & 0.6 \\
\hline \multicolumn{8}{|l|}{ Gender } \\
\hline Female & 110 & 37 & Ref. & & 55 & Ref. & \\
\hline Male & 390 & 128 & $0.98(0.58-1.69)$ & 0.96 & 171 & $1.13(0.72-1.79)$ & 0.6 \\
\hline \multicolumn{8}{|l|}{ Smoking } \\
\hline Ever & 154 & 51 & Ref. & & 76 & Ref. & \\
\hline Never & 346 & 114 & $1.00(0.62-1.63)$ & 0.99 & 150 & $0.78(0.51-1.17)$ & 0.23 \\
\hline \multicolumn{8}{|l|}{ Histology } \\
\hline Squamous carcinoma & 268 & 84 & Ref. & & 113 & Ref. & \\
\hline Adenocarcinoma & 146 & 35 & $0.60(0.39-0.92)$ & 0.02 & 59 & $0.77(0.54-1.09)$ & 0.14 \\
\hline Othersa & 86 & 46 & $1.43(0.88-2.33)$ & 0.15 & 54 & $1.19(0.78-1.83)$ & 0.42 \\
\hline \multicolumn{8}{|l|}{ TNM stage } \\
\hline I & 119 & 27 & Ref. & & 37 & Ref. & \\
\hline II & 162 & 46 & $1.47(0.88-2.44)$ & 0.14 & 62 & $1.30(0.86-1.96)$ & 0.21 \\
\hline III & 199 & 82 & $2.26(1.42-3.58)$ & $<0.01$ & 114 & $2.31(1.58-3.37)$ & $<0.01$ \\
\hline IV & 20 & 10 & $3.03(1.41-6.52)$ & $<0.01$ & 13 & $2.61(1.35-5.05)$ & $<0.01$ \\
\hline \multicolumn{8}{|l|}{ Differentiation } \\
\hline Well & 25 & 6 & Ref. & & 9 & Ref. & \\
\hline Moderate & 316 & 83 & $1.00(0.42-2.37)$ & 1 & 120 & $1.08(0.53-2.18)$ & 0.83 \\
\hline Poor & 159 & 76 & $1.59(0.66-3.86)$ & 0.3 & 97 & $1.80(0.86-3.73)$ & 0.12 \\
\hline \multicolumn{8}{|c|}{ Adjuvant chemo-or radiotherapy } \\
\hline No & 165 & 85 & Ref. & & 93 & Ref. & \\
\hline Yes & 335 & 80 & $0.64(0.44-0.92)$ & 0.02 & 132 & $0.81(0.58-1.12)$ & 0.21 \\
\hline
\end{tabular}


Cox regression model (Table 3). Our data showed that two SNPs (rs4246444 and rs4485435) in FASN gene were significantly associated with NSCLC recurrence. SNP rs4246444 exhibited a significant association with recurrence in NSCLC in the additive model (HR, 0.82 ; 95\% CI, 0.67-1.00; $p=0.05$ ). Under the dominant model, rs4485435 exhibited significant associations with recurrence $(\mathrm{HR}, 0.75 ; 95 \% \mathrm{CI}, 0.56-1.01 ; p=0.05)$. In addition, SNP rs 9912300 in ACLY gene was significantly associated with death risk (HR, 1.41; 95\% CI, 1.02-1.94; $p=0.04$ ). No negative association was observed between the other five SNPs and lung cancer patient outcomes, although four of them were located in exons. Q values of the four positive SNPs indicated a relative low probability of false-positive results (all Q values <0.10). Furthermore, Kaplan-Meier curve analysis demonstrated significant or borderline significant differences between patient groups by SNPs under the best fitting model in OS and recurrencefree survival (RFS) analyses (Figure 1).

Cumulative effect of unfavorable genotypes on prognosis of NSCLC patients

In order to assess the cumulative effects of genetic variants on lung cancer outcomes, we did a joint analysis by including the four unfavorable genotypes determined by the single SNP analysis (Table 4). Briefly, the unfavorable genotypes were homozygous variant TT for rs4246444, homozygous variant GG for rs4485435, and combination of TG and GG for rs9912300. Patients were

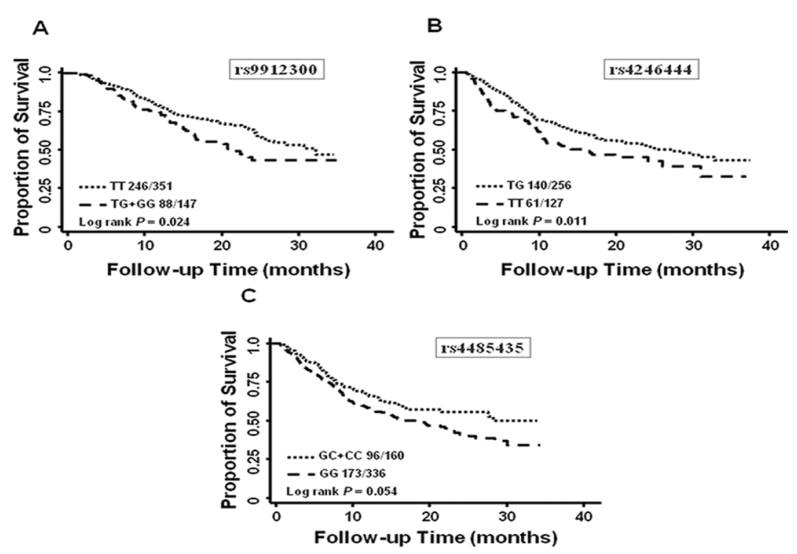

Figure 1. Kaplan-Meier Curves of Overall. (A) and recurrence-free $(B, C)$ survival of NSCLC patients stratified by SNPs

Table 3. Association of single SNPs in DNL genes with clinical outcome of non-small cell lung cancer patients

\begin{tabular}{|c|c|c|c|c|c|c|c|c|c|}
\hline \multirow{2}{*}{$\begin{array}{r}\text { Gene/SNP } \\
\text { be }\end{array}$} & \multirow{2}{*}{$\begin{array}{c}\text { Genotype and } \\
\text { pest fitting model }\end{array}$} & \multicolumn{4}{|c|}{ Overall survival } & \multicolumn{4}{|c|}{ Recurrence-free survival } \\
\hline & & Death/Total & $\mathrm{HR}^{\mathrm{a}}(95 \% \mathrm{CI})$ & $\mathrm{p}^{\mathrm{a}}$ & $\bar{Q}^{\mathrm{b}}$ & Recurrence/ Total & $\mathrm{HR}^{\mathrm{a}}(95 \% \mathrm{CI})$ & $\mathrm{p}^{\mathrm{a}}$ & $\mathrm{Q}^{\mathrm{b}}$ \\
\hline \multicolumn{10}{|l|}{ ACACA/ } \\
\hline \multirow{4}{*}{ rs1714987 } & GG & $44 / 143$ & Ref. & & & $63 / 143$ & Ref. & & \\
\hline & GC & $78 / 250$ & $1.03(0.71-1.49)$ & & & $106 / 250$ & $1.04(0.76-1.41)$ & & \\
\hline & $\mathrm{CC}$ & $42 / 105$ & $1.19(0.77-1.83)$ & & & $55 / 105$ & $1.12(0.78-1.62)$ & & \\
\hline & Additive & & $1.09(0.88-1.36)$ & 0.44 & 0.23 & & $1.06(0.88-1.27)$ & 0.54 & 0.23 \\
\hline \multirow[t]{4}{*}{ rs11871275 } & $\mathrm{AA}$ & $105 / 350$ & Ref. & & & $150 / 350$ & Ref. & & \\
\hline & AT & $55 / 134$ & $1.32(0.95-1.85)$ & & & $66 / 134$ & $1.12(0.84-1.50)$ & & \\
\hline & TT & $4 / 14$ & $0.64(0.23-1.76)$ & & & $8 / 14$ & $0.89(0.43-1.84)$ & & \\
\hline & Additive & & $1.10(0.84-1.44)$ & 0.5 & 0.23 & & $1.05(0.85-1.33)$ & 0.7 & 0.25 \\
\hline \multirow[t]{4}{*}{ rs7211875 } & $\mathrm{TT}$ & $90 / 263$ & Ref. & & & $120 / 263$ & Ref. & & \\
\hline & $\mathrm{TC}$ & $57 / 197$ & $0.86(0.62-1.21)$ & & & $82 / 197$ & $0.95(0.72-1.26)$ & & \\
\hline & $\mathrm{CC}$ & $17 / 38$ & $1.39(0.82-2.37)$ & & & $22 / 38$ & $1.43(0.91-2.25)$ & & \\
\hline & Additive & & $1.05(0.82-1.34)$ & 0.72 & 0.25 & & $1.09(0.89-1.34)$ & 0.41 & 0.23 \\
\hline \multicolumn{10}{|l|}{ ACLY/ } \\
\hline \multirow[t]{4}{*}{ rs2304497 } & TT & $141 / 439$ & Ref. & & & $197 / 439$ & Ref. & & \\
\hline & TG & $22 / 56$ & $1.26(0.80-1.99)$ & & & $25 / 56$ & $1.02(0.68-1.53)$ & & \\
\hline & GG & $1 / 3$ & $2.39(0.33-17.47)$ & & & $2 / 3$ & $4.17(1.01-17.28)$ & & \\
\hline & Additive & & $1.30(0.85-1.99)$ & 0.22 & 0.22 & & $1.14(0.78-1.65)$ & 0.5 & 0.23 \\
\hline \multirow[t]{4}{*}{ rs9912300 } & $\mathrm{TT}$ & $105 / 351$ & Ref. & & & $154 / 351$ & Ref. & & \\
\hline & TG & $55 / 136$ & $1.46(1.05-2.02)$ & & & $63 / 135$ & $1.05(0.79-1.40)$ & & \\
\hline & GG & $2 / 11$ & $0.70(0.17-2.87)$ & & & $6 / 11$ & $1.53(0.67-3.53)$ & & \\
\hline & Dominant & & $1.41(1.02-1.94)$ & 0.04 & 0.09 & & $1.10(0.85-1.42)$ & 0.46 & 0.23 \\
\hline \multicolumn{10}{|l|}{ FASN/ } \\
\hline \multirow[t]{4}{*}{ rs4246444 } & TT & $47 / 127$ & Ref. & & & $66 / 127$ & Ref. & & \\
\hline & TG & $83 / 256$ & $0.91(0.63-1.31)$ & & & $116 / 256$ & $0.85(0.63-1.16)$ & & \\
\hline & GG & 30/109 & $0.76(0.48-1.22)$ & & & $37 / 109$ & $0.66(0.44-1.00)$ & & \\
\hline & Additive & & $0.88(0.70-1.10)$ & 0.26 & 0.22 & Additive & $0.82(0.67-1.00)$ & 0.05 & 0.09 \\
\hline \multirow[t]{4}{*}{ rs1140616 } & $\mathrm{CC}$ & $75 / 213$ & Ref. & & & $106 / 213$ & Ref. & & \\
\hline & $\mathrm{CT}$ & $70 / 227$ & $0.91(0.66-1.27)$ & & & $93 / 227$ & $0.83(0.63-1.10)$ & & \\
\hline & $\mathrm{TT}$ & $19 / 54$ & $1.09(0.65-1.82)$ & & & $24 / 54$ & $1.05(0.67-1.65)$ & & \\
\hline & Additive & & $1.00(0.79-1.26)$ & 0.98 & 0.32 & & $0.95(0.77-1.17)$ & 0.62 & 0.25 \\
\hline \multirow[t]{4}{*}{ rs4485435 } & GG & $122 / 336$ & Ref. & & & $163 / 336$ & Ref. & & \\
\hline & GC & $36 / 144$ & $0.70(0.48-1.01)$ & & & $54 / 144$ & $0.71(0.52-0.97)$ & & \\
\hline & $\mathrm{CC}$ & $6 / 12$ & $1.40(0.61-3.22)$ & & & $6 / 12$ & $1.24(0.54-2.82)$ & & \\
\hline & Dominant & & $0.75(0.53-1.07)$ & 0.11 & 0.13 & Dominant & $0.75(0.56-1.01)$ & 0.05 & 0.09 \\
\hline
\end{tabular}

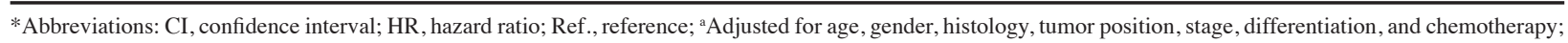
${ }^{\mathrm{b}} \mathrm{Q}$ value, multiple comparison adjustment by $\mathrm{Q}$ test 
Table 4. Cumulative Effects of Unfavorable Genotypes on Prognosis of Non-small cell lung cancer patients

\begin{tabular}{|c|c|c|c|c|c|c|}
\hline $\begin{array}{l}\text { Group (number of } \\
\text { unfavorable genotypes }{ }^{\mathrm{a}} \text { ) }\end{array}$ & Death/total & $\begin{array}{l}\text { Overall survival } \\
\operatorname{HR}^{\mathrm{b}}(95 \% \mathrm{CI})\end{array}$ & $P$ & Recurrence/total & $\begin{array}{l}\text { Recurrence-free survival } \\
\operatorname{HR}^{\mathrm{b}}(95 \% \mathrm{CI})\end{array}$ & $P$ \\
\hline Group $1(0)$ & $22 / 104$ & Ref. & & $36 / 104$ & Ref. & \\
\hline Group 2 (1) & $64 / 198$ & $1.55(0.95-2.52)$ & 0.08 & $88 / 198$ & $1.41(0.95-2.09)$ & 0.09 \\
\hline Group $3(\geq 2)$ & $74 / 185$ & $1.83(1.13-2.96)$ & 0.01 & $94 / 185$ & $1.54(1.04-2.28)$ & 0.03 \\
\hline$P$ value for trend & & & 0.02 & & & 0.04 \\
\hline
\end{tabular}

*Abbreviations: CI, confidence interval; HR, hazard ratio; Ref., reference; a Unfavorable genotypes: FASN rs4246444 (TT), rs4485435 (GG), and ACLY rs9912300 $(\mathrm{TG}+\mathrm{GG}) ;{ }^{\text {b}}$ Adjusted for age, sex, smoking status, histological type, stage, differentiation, and chemotherapy
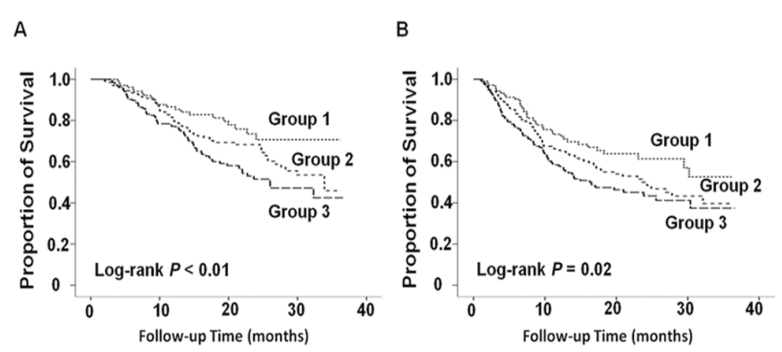

Figure 2. The Cumulative Effects of Unfavorable Genotypes of FASN and ACLY Genes on Overall. (A) and recurrence-free (B) survival of NSCLC patients analyzed by Kaplan-Meier curves. Group 1, patients with no unfavorable genotype; Group 2, patients with 1 unfavorable genotype; Group 3 , patients with at least 2 unfavorable genotypes

divided into three groups according to the number of unfavorable genotypes they carried. Compared with those of group 1 ( 0 unfavorable genotype), there was a 1.41-fold increased risk of recurrence $(95 \% \mathrm{CI}, 0.95-2.09 ; p=0.09)$ in patients of group 2 ( 1 unfavorable genotype), and a 1.54-fold increased risk of recurrence (95\% CI, 1.04-2.28; $p=0.03$ ) in patients of group 3 (with at least 2 unfavorable genotypes), with a significant moderate dose-dependent effect among the three groups ( $\mathrm{P}$ for trend $=0.04$ ). Similar results were also found for OS. Group 2 patients had a 1.55-fold increased risk of death (95\% CI, 0.95-2.52, $p=0.08$ ), and group 3 had a 1.83 -fold increase in risk of death (95\%CI, 1.13-2.96, $p=0.01$ ), when comparing with group 1 ( $\mathrm{P}$ for trend=0.02). Kaplan-Meier plots also showed the significantly different RFS and OS proportions among the three groups over the follow-up (Figure. 2).

\section{Discussion}

In the current study, we evaluated the effects of eight functional SNPs in three genes (ACACA, FASN, and ACLY) of DNL pathway on the prognosis of NSCLC patients. We found that two SNPs (rs4246444 and rs4485435) located in the FASN gene were significantly associated with the risk of NSCLC recurrence after surgery. In addition, SNP rs9912300 in ACLY gene was significantly associated with OS of NSCLC patients. Furthermore, we identified an accumulative risk of death with increasing number of unfavorable genotypes. To the best of our knowledge, this is the first study to report that genetic variants in the DNL genes have a significant effect on the prognosis in NSCLC patients.

Increased DNL has been considered as a hallmark of cancer, whereas its significance in cancer pathogenesis has long been underestimated. Recent studies have shown the importance of the lipogenic enzymes in survival, proliferation, progression, angiogenesis and drug resistance of cancer cells. (Swinnen et al., 2006) It has been demonstrated that FASN gene is over-expressed in various types of cancer, including NSCLC, under the driving of growth factor signaling pathway, including the phosphatidylinositol-3-kinase (PI3K)-Akt and mitogen-activated protein kinase (MAPK) pathway (Li et al., 2012b). Treating tumor cells with pharmacological inhibitors of FASN or silencing FASN expression by specific siRNAs effectively suppresses the growth and induces apoptosis in cancer cells both in vitro and in vivo (Pizer et al., 2000; De Schrijver et al., 2003). In addition, it has been reported that inhibition of FASN expression significantly suppressed the invasion and radiation resistance in cancer cells (Yang et al., 2011).

ACLY catalyzes the conversion of citrate to cytosolic acetyl-CoA, thus linking the tumor-associated increase in glycolysis to enhanced lipogenesis. Similar as FASN and ACACA, marked elevation of ACLY expression and activity has been reported in cancer cells (Bauer et al., 2005; Hatzivassiliou et al., 2005). In human lung adenocarcinoma, the expression of phosphorylated ACLY is correlated with stage, differentiation grade, and a poorer prognosis. Moreover (Hatzivassiliou et al., 2005), ACLY inhibition by siRNAs or the selective inhibitor SB-204990 suppresses the growth and survival of tumor cells in vitro and in vivo (Bauer et al., 2005; Hatzivassiliou et al., 2005; Migita et al., 2008). Although the molecular mechanisms of ACLY inhibition-dependent cell death are still not clear, these data suggest that this enzyme could also be a target for cancer therapy.

Genetic variants such as SNPs play an important role in the regulation of gene expression, mRNA degradation and translation, as well as protein structures which may affect the biological functions of proteins (Shastry, 2009). Considering the critical role of DNL genes in the development of cancer, effects of SNPs in these genes on cancer cell proliferation, invasion and drug sensitivity as well as clinicopathologcal characteristics and prognosis of patients should be investigated. However, little is known on the associations between these SNPs and cancer risk as well as patient prognosis. It has been reported that SNP rs4247357 in the FASN gene is associated with the risk of uterine leiomyomata (Eggert et al., 2012). In addition, Nguyen PL et al have reported that SNP rs1127678 in the FASN gene is associated with the risk and mortality of prostate cancer (Nguyen et al., 2010). In line with these findings, we found that two SNPs (rs4246444 and rs4485435) in FASN gene were significantly associated with the risk of recurrence in NSCLC patients, while SNP rs9912300 in ACLY gene was associated with the OS of 
NSCLC patients. These data suggest that different SNPs in DNL genes might affect the risk or prognosis of different tumors. However, the differences in the biological functions of these SNPs need to be further investigated.

Although the molecular and cellular functions of these SNPs have not yet been evaluated, silicon analysis found that rs4485435 in the exon 21 of FASN may affect alternative splicing of mRNA. The study of Dolley et al has suggested that rs4246444 is a potential functional SNP, although it is located in intron of FASN (Dolley et al., 2011). MultiZ alignment has estimated that none of the three FASN SNPs is located in the highly conserved regions of the genome (Blanchette et al., 2004). SNP rs9912300 is located in the intron region of ACLY, and is a translation factor binding site-related SNP. Further functional research on these SNPs is needed to elucidate their effects on gene expression, DNL activity as well as patient prognosis in NSCLC.

Presence of multiple SNPs had a cumulative effect on recurrence and survival of lung cancer patients. Studying the cumulative effects may lead to more powerful prognostic or predictive signatures based on the combination of each patient's genetic variations. The present study demonstrated a significant dosedependent effect of the DNL gene variants on the survival and recurrence of lung cancer patients. Hence, more unfavorable genotypes indicate poorer prognosis for lung cancer patients. It should be noted that this study was exploratory, and the results need to be validated in independent studies.

Our study has a few strengths. First, our pathwaybased approach is a logical extension of the candidate gene approach and avoids the requirement of a much larger sample size for a genome-wide association study. Secondly, the patient cohort in this study was relatively large and recruited from a single institution. The uniform standard operation procedures in cancer identification, pathological staging, and cancer treatment strategy make our findings more comprehensive and applicable to future clinical studies. Further, the cumulative effects analysis established a novel combination of SNPs to predict the outcome of lung cancer patients, which could be investigated further and included in a prognostic model to help clinicians in predicting outcomes for lung cancer patients. Nevertheless, some limitations also exist in our study, such as lacking of generalizability, unclear biological mechanisms of the significant SNPs, and a relatively smaller sample size in stratified analysis. Further validation on our results in populations with other ethnicities is needed to generalize the potential clinical utility of our findings.

Overall, as the first study observing the effect of DNL gene polymorphisms on NSCLC prognosis in a Chinese population, our results strongly suggest that certain genetic variants of FASN and ACLY genes may be independent prognostic markers for recurrence and survival prediction in NSCLC patients after resection. These findings indicate that further studies are warrant edon the impact of these SNPs on the effectiveness of therapeutics against DNL in NSCLC.

\section{Acknowledgements}

The authors declare that they have no conflict of interest. This work was supported by Program for New Century Excellent Talents in University, National Natural Science Foundation (81171966) and National Key Technologies R\&D Program (2011ZX09307-001-04) of China.

\section{References}

Alo PL, Visca P, Marci A, et al (1996). Expression of fatty acid synthase (FAS) as a predictor of recurrence in stage I breast carcinoma patients. Cancer, 77, 474-82.

Bauer DE, Hatzivassiliou G, Zhao F, et al (2005). ATP citrate lyase is an important component of cell growth and transformation. Oncogene, 24, 6314-22.

Blanchette M, Kent WJ, Riemer C, et al (2004). Aligning multiple genomic sequences with the threaded blockset aligner. Genome Res, 14, 708-15.

Cantor JR, and Sabatini DM (2012). Cancer cell metabolism: one hallmark, many faces. Cancer Discov, 2, 881-98.

Cerne D, Zitnik IP, Sok M (2010). Increased fatty acid synthase activity in non-small cell lung cancer tissue is a weaker predictor of shorter patient survival than increased lipoprotein lipase activity. Arch Med Res, 41, 405-9.

Chajes V, Cambot M, Moreau K, et al (2006). Acetyl-CoA carboxylase alpha is essential to breast cancer cell survival. Cancer Res, 66, 5287-94.

De Schrijver E, Brusselmans K, Heyns W, et al (2003). RNA interference-mediated silencing of the fatty acid synthase gene attenuates growth and induces morphological changes and apoptosis of $\mathrm{LNCaP}$ prostate cancer cells. Cancer Res, 63, 3799-804.

Dinse GE, Lagakos SW (1982). Nonparametric estimation of lifetime and disease onset distributions from incomplete observations. Biometrics, 38, 921-32.

Dolley G, Boisclair ME, Lamarche B, et al (2011). Interactions between dietary fat intake and FASN genetic variation influence LDL peak particle diameter. J Nutrigenet Nutrige, 4, 137-45.

Eggert SL, Huyck KL, Somasundaram P, et al (2012). Genomewide linkage and association analyses implicate FASN in predisposition to Uterine Leiomyomata. Am J Hum Genet, 91, 621-8.

Flynn R (2012). Survival analysis. J Clin Nurs, 21, 2789-97.

Goldstraw P, Ball D, Jett JR, et al (2011). Non-small-cell lung cancer. Lancet, 378, 1727-40.

Hatzivassiliou G, Zhao F, Bauer DE, et al (2005). ATP citrate lyase inhibition can suppress tumor cell growth. Cancer Cell, 8, 311-21.

Jemal A, Bray F, Center MM, et al (2011). Global cancer statistics. CA-Cancer J Clin, 61, 69-90.

Kao YC, Lee SW, Lin LC, et al (2013). Fatty acid synthase overexpression confers an independent prognosticator and associates with radiation resistance in nasopharyngeal carcinoma. Tumor Biol, 34, 759-68.

Kuhajda FP (2000). Fatty-acid synthase and human cancer: new perspectives on its role in tumor biology. Nutrition, 16, 202-8.

Laing RE, Hess P, Shen Y, et al (2011). The role and impact of SNPs in pharmacogenomics and personalized medicine. Curr Drug Metab, 12, 460-86.

Li N, Bu X, Tian X, et al (2012a). Fatty acid synthase regulates proliferation and migration of colorectal cancer cells via 
HER2-PI3K/Akt signaling pathway. Nutr Cancer, 64, 864-70.

Li N, Bu X, Wu P, et al (2012b). The "HER2-PI3K/Akt-FASN Axis" regulated malignant phenotype of colorectal cancer cells. Lipids, 47, 403-11.

Lin R, Tao R, Gao X, et al (2013). Acetylation stabilizes ATPcitrate lyase to promote lipid biosynthesis and tumor growth. Mol Cell, 51, 506-18.

Liu HB, Peng YP, Dou CW, et al (2012). Comprehensive study on associations between nine SNPs and glioma risk. Asian Pac J Cancer Prev, 13, 4905-8.

Migita T, Narita T, Nomura K, et al (2008). ATP citrate lyase: activation and therapeutic implications in non-small cell lung cancer. Cancer Res, 68, 8547-54.

Migita T, Ruiz S, Fornari A, et al (2009). Fatty acid synthase: a metabolic enzyme and candidate oncogene in prostate cancer. J Natl Cancer Inst, 101, 519-32.

Nguyen PL, Ma J, Chavarro JE, et al (2010). Fatty acid synthase polymorphisms, tumor expression, body mass index, prostate cancer risk, and survival. J Clin Oncol, 28, 3958-64.

Ogino S, Nosho K, Meyerhardt JA, et al (2008). Cohort study of fatty acid synthase expression and patient survival in colon cancer. J Clin Oncol, 26, 5713-20.

Pizer ES, Thupari J, Han WF, et al (2000). Malonyl-coenzyme-A is a potential mediator of cytotoxicity induced by fattyacid synthase inhibition in human breast cancer cells and xenografts. Cancer Res, 60, 213-8.

Rahman MT, Nakayama K, Rahman M, et al (2012). Fatty acid synthase expression associated with NAC1 is a potential therapeutic target in ovarian clear cell carcinomas. Brit $J$ Cancer, 107, 300-7.

Shastry BS (2009). SNPs: impact on gene function and phenotype. Meth Mol Biol, 578, 3-22.

Shurbaji MS, Kalbfleisch JH, Thurmond TS (1996). Immunohistochemical detection of a fatty acid synthase (OA-519) as a predictor of progression of prostate cancer. Hum Pathol, 27, 917-21.

Storey JD, Taylor JE, Siegmund D (2004). Strong control, conservative point estimation and simultaneous conservative consistency of false discovery rates: a unified approach. $J R$ Stat Soc Series B Stat Methodol, 66, 187-205.

Swinnen JV, Brusselmans K, Verhoeven G (2006). Increased lipogenesis in cancer cells: new players, novel targets. Curr Opin Clin Nutr, 9, 358-65.

Wang Y, Wang Y, Shen L, et al (2012). Prognostic and therapeutic implications of increased ATP citrate lyase expression in human epithelial ovarian cancer. Oncol Rep, 27, 1156-62.

Warburg O (1956). On the origin of cancer cells. Science, 123, 309-14.

Xing J, Myers RE, He X, et al (2011). GWAS-identified colorectal cancer susceptibility locus associates with disease prognosis. Eur J Cancer, 47, 1699-707.

Yang Y, Liu H, Li Z, et al (2011). Role of fatty acid synthase in gemcitabine and radiation resistance of pancreatic cancers. Int J Biochem Mol Biol, 2, 89-98.

Zhou F, He X, Liu H, et al (2012). Functional polymorphisms of circadian positive feedback regulation genes and clinical outcome of Chinese patients with resected colorectal cancer. Cancer, 118, 937-46. 